\title{
LORENTZIAN MANIFOLDS OF NONPOSITIVE CURVATURE. II
}

\author{
F. J. FLAHERTY
}

\begin{abstract}
Suppose that $M$ is a time oriented, future 1-connected, timelike and null geodesically complete Lorentzian manifold. Previously, we have shown the exponential map at any point of such a manifold embeds the future cone into $M$ when $M$ has nonpositive spacetime curvatures. Here we want to demonstrate that under the same hypotheses, $M$ is homeomorphic to the product of the real line with a Cauchy hypersurface.
\end{abstract}

Let us recall briefly the main points in $[F]$. The basic object of study is a Lorentzian $n$-manifold $M$ (signature $2-n$ ), which we suppose time orientable. We tacitly assume then that $M$ is time oriented. The manifold $M$ is called future 1-connected iff any two future-timelike (smooth) curves from $p$ to $q$ are homotopic through future-timelike curves with endpoints $p$ and $q$. The spacetime curvatures of $M$ are the sectional curvatures of planes spanned by a timelike and a spacelike vector. We now state Proposition 2.1 of $[\mathbf{F}]$ : Let $M$ be a time oriented Lorentzian manifold with nonpositive spacetime curvatures; then the exponential map at any point of $M$ has maximal rank on the (closed) future cone. This proposition is very useful in proving the main theorem of $[\mathbf{F}]$, which is stated here in the first paragraph.

Briefly our intention is to introduce the notion of a globally hyperbolic manifold, much the same as Leray did in [L], and prove that our manifolds are globally hyperbolic. Our theorem then follows from a result of Geroch, that globally hyperbolic spaces are homeomorphic to a product of the real line with a Cauchy hypersurface [G].

The first problem is to extend the ideas of timelike or null - defined only for smooth curves-to continuous curves. We use essentially the definition of Hawking and Ellis [HE] for nonspacelike curves. A continuous curve $c$ mapping an interval of real numbers $I$ into $M$ is called a nonspacelike curve iff for any $t$ in $I$ there is an $\eta>0$ and a normal neighborhood $U$ of

Presented to the Society, January 15, 1974; received by the editors December 7 , 1973 and, in revised form, January 17, 1974.

AMS (MOS) subject classifications (1970). Primary 53B 30; Secondary 83F05.

Key words and phrases. Future 1-connected, nonspacelike curves, exponential map, spacetime curvature, globally hyperbolic. 
$c(t)$ such that for $t+\eta>s>t$ there is a future-timelike or -null curve joining $c(s)$ to $c(t)$ in $U$, and if $t-\eta<s<t$, there is a past-timelike or -null curve joining $c(s)$ to $c(t)$ in $U$. Intuitively nonspacelike curves are continuous timelike or null curves. A more thorough discussion can be found in $[\mathbf{G}]$ or [HE].

Let $C(p, q)$ denote the set of all equivalence classes of nonspacelike curves from $p$ to $q$ under the relation of reparameterization by continuous monotonic maps. Provide $C(p, q)$ with the compact open topology and observe that

$$
C(p, q)=\overline{C^{+}(p, q)} \cup \Omega_{0}(p, q),
$$

where $C^{+}(p, q)$ is the space of timelike curves from $p$ to $q$ and $\Omega_{0}(p, q)$ is the space of unbroken null geodesics from $p$ to $q$ without conjugate points. This follows from [HE, §4.5]. Following Leray, a time oriented manifold $M$ is said to be globally hyperbolic iff $C(p, q)$ is empty or compact for all $p$ and $q$ in $M$. Geroch gives a geometric way of looking at the convergence of curves in $C(p, q)$ when there are no closed nonspacelike curves, compare [G].

Theorem. Let $M$ be a future 1-connected manifold which is timelike and null geodesically complete. Further suppose that the spacetime curvatures of $M$ are nonpositive. Then $M$ is globally hyperbolic.

Proof. Suppose that $C(p, q)$ is nonempty. If $C^{+}(p, q)$ is empty, then $C(p, q)=\Omega_{0}(p, q)$. Let $N_{0}$ be the set of all null vectors $u$ such that $c(t)=$ $\exp (t u)$ is in $\Omega_{0}(p, q)$. Clearly $N_{0}$ is a discrete set because exp has maximal rank at each $u$ in $N_{0}$ by Proposition 2.1 of [F]. Now if $N_{0}$ were an unbounded set, we could find a sequence $\left(u_{n}\right)$ from $N_{0}$ such that $u_{n} \rightarrow \infty$. So, given neighborhoods $U_{n}$ of radius $1 / n$ around $q$, we can find, by continuity, neighborhoods $V_{n}$ of $u_{n}$ such that $\exp \left(V_{n}\right) \subset U_{n}$. If we take a sequence of timelike vectors $v_{n}$ in $V_{n}$, then it follows that $\exp \left(v_{n}\right)=q_{n} \rightarrow q$. In addition, $v_{n}$ can be chosen arbitrarily close to $u_{n}$ and so $v_{n} \rightarrow \infty$ as well. Again from Proposition 2.1 of [F], it follows that exp is of maximal rank at $u_{1}$, $\exp \left(u_{1}\right)=q$, and so there are compact neighborhoods $U$ of $q$ and $V$ of $u_{1}$ such that $\exp$ maps $V$ diffeomorphically onto $U$. Further, there is a positive integer $n_{0}$ for which $n \geq n_{0}$ implies $q_{n}$ is in $U$. From the main theorem of $[\mathbf{F}]$, exp has an inverse on the set of timelike vectors, and the restriction of this inverse to the image of timelike vectors in $V$ must agree with the inverse of the map $\exp _{V}$ (restriction to $V$ ) on the image of timelike vectors 
in $V$. Hence

$$
\exp ^{-1}\left(q_{n}\right)=\exp ^{-1}\left(\exp \left(v_{n}\right)\right)=v_{n}
$$

for $n \geq n_{0}$; so the $v_{n}$ are in $V$, contradicting the fact that $v_{n} \rightarrow \infty$. As a result $u_{n}$ does not go to infinity, and thus the set $N_{0}$ is bounded and so finite. In this case $C(p, q)$ is compact. If $C^{+}(p, q)$ were nonempty, $q=$ $\exp (u)$ for some timelike $u$ (again by the main theorem of [F]). First we want to prove that $\exp : C(0, u) \rightarrow C^{+}(p, q)$ is onto, where $C(0, u)$ is the set of nonspacelike curves in the tangentspace from 0 to $u$. Thus for $c=$ $\lim c_{n}, c_{n}$ in $C^{+}(p, q)$, the curves $a_{n}=\exp ^{-1} c_{n}$ are timelike curves in the tangentspace, by a similar argument as in the proof of the main theorem of $[F]$. It follows from the compactness of $C(0, u)$ that $a_{n} \rightarrow a$, possibly passing to a subsequence, and since exp is defined on the closed cone, $\exp (a)$ makes sense. Moreover the map exp: $C(0, u) \rightarrow C(p, q)$ is continuous in the compact open topology so $\exp (a)=c$. Again $\Omega_{0}(p, q)$ is the image of a finite set, so $C(p, q)$ is the continuous image of a compact set and, hence, compact.

Finally, we state Geroch's result on globally hyperbolic manifolds. First, a subset $S$ of a Lorentzian manifold is called achronal iff no $p$ and $q$ in $S$ can be joined by a timelike curve. Now define $D^{+}(S)$ (respectively $D^{-}(S)$ ) to be the set of points $p$ such that every past (respectively future) directed timelike curve from $p$ without a past (respectively future) endpoint intersects $S$. An achronal subset $S$ of $M$ is called a Cauchy hypersurface iff $D^{+}(S) \cup D^{-}(S)=M$.

Theorem [G]. $M$ is globally byperbolic iff $M$ contains a Cauchy hypersurface $S$, in which case $M$ is homeomorphic to the product of the real line with $S$.

Theorem. Let $M$ be a future 1-connected manifold which is timelike and null geodesically complete. Further suppose that the spacetime curvatures of $M$ are nonpositive. Then $M$ is homeomorphic to the product of the real line with a Cauchy hypersurface.

In conclusion, let us give an example of a Lorentzian manifold that is simply connected but not future 1-connected. This example is due to R. P. Geroch. Consider ordinary Minkowski three-space with coordinates $x, y$, and $t$. Let $U$ be the open set $|t|<1$ with the positive $x$-axis removed as well as the interval from -2 to 2 on the $y$-axis. The union of the removed 
sets is an infinite $T$-shaped figure. The set $U$ is clearly simply connected. Choose points $p$ and $q$ above and below the $x$-axis, respectively, and join them by timelike curves straddling the $x$-axis. These two curves cannot be homotoped by timelike curves, since you would have to go around the part of the $y$-axis that has been excluded, which is impossible without introducing spacelike curves. Avez has considered a similar notion of timelike homotopy in $[A]$ as has J. W. Smith in [S].

Finally it would be interesting to prove this theorem with future 1-connected replaced by simply connected.

\section{REFERENCES}

[A] A. Avez, Essais de géométrie riemannienne hyperbolique-applications à la relativité générale, Ann. Inst. Fourier (Grenoble) 13 (1963), 105-190.

[F] F. J. Flaherty, Lorentzian manifolds of non-positive curvature, Proc. Sympos。 Pure Math., vol。27, Amer。Math.Soc., Providence, R。I. (to appear).

[G] R. P. Geroch, Domain of dependence, J. Mathematical Phys. 11 (1970), 437-449. MR $42 \# 5585$.

[HE] S. W. Hawking and G. F. R. Ellis, The large scale structure of space time, Cambridge Monographs in Mathematical Physics, Cambridge, 1973.

[L] J. Leray, Hyperbolic differential equations, Institute for Advanced Study, Princeton, N. J., 1953. MR 16, 139.

[S] J. W. Smith, Fundamental groups on a Lorentz manifold, Amer. J. Math. 82 (1960), 873-890. MR $22 \# 11350$. GON 97331

DEPARTMENT OF MATHEMATICS, OREGON STATE UNIVERSITY, CORVALLIS, ORE• 